\title{
UNIONS OF JOHN DOMAINS AND UNIFORM DOMAINS IN REAL NORMED VECTOR SPACES
}

\author{
Yaxiang Li and Xiantao Wang* \\ Hunan Normal University, Department of Mathematics \\ Changsha, Hunan 410081, P. R. China; yaxiangli@163.com \\ Hunan Normal University, Department of Mathematics \\ Changsha, Hunan 410081, P. R. China; xtwang@hunnu.edu.cn
}

\begin{abstract}
Let $E$ be real normed vector spaces with the dimension at least 2. In this paper we study the following questions: When is the union of two John domains in $E$ a John domain and when is the union of two uniform domains in $E$ a uniform domain?
\end{abstract}

\section{Introduction and main results}

Throughout the paper, we always assume that $E$ denotes a real normed vector space with $\operatorname{dim} E \geq 2$ and that $D$ is a proper subdomain in $E$. The norm of a vector $z$ in $E$ is written as $|z|$, and for any two points $z_{1}, z_{2}$ in $E$, the distance between them is denoted by $\left|z_{1}-z_{2}\right|$, and the closed line segment with endpoints $z_{1}$ and $z_{2}$ by $\left[z_{1}, z_{2}\right]$. For $x \in E$ and $r>0$, we let $\mathbf{B}(x, r)$ denote the open ball in $E$ with center $x$ and radius $r$. For real numbers $r$ and $s$, we use the notation: $r \wedge s=\min \{r, s\}$.

John domains in Euclidean spaces $\mathbf{R}^{n}$ were introduced by John [1] in connection with his work on elasticity. The term is due to Martio and Sarvas [3]. Roughly speaking, a domain is a John domain if it is possible to travel from one point of the domain to another without going too close to the boundary. The precise definition is as follows.

Definition 1.1. $D$ is called a $c$-John domain if for every pair of points $x_{1}, x_{2} \in D$ there is a rectifiable arc $\gamma$ joining them with

$$
\ell\left(\gamma\left[x_{1}, x\right]\right) \wedge \ell\left(\gamma\left[x_{2}, x\right]\right) \leq c d(x)
$$

for all $x \in \gamma$, where $c$ is a positive constant, $\gamma\left[x_{j}, x\right]$ denotes the closed subarc of $\gamma$ with endpoints $x_{j}$ and $x(j=1,2), \ell\left(\gamma\left[x_{j}, x\right]\right)$ the arclength of $\gamma\left[x_{j}, x\right] . \gamma$ is called a c-John arc joining $x_{1}$ and $x_{2}$.

See [4] for several characterizations of John domains. In the study of John domains, the following question is natural:

Question 1.1. Is the union of two John domains in E still a John domain when their intersection is not empty?

Väisälä considered this question when $E=\mathbf{R}^{n}$. In [7], Väisälä constructed an example to show that, in general, the answer to this question is negative. Note

doi:10.5186/aasfm.2010.3539

2000 Mathematics Subject Classification: Primary 30C65, 30C45; Secondary 30C20.

Key words: Union, John domain, uniform domain, bounded and convex domain.

* Corresponding author.

The research was partly supported by NSFs of China (No. 10771059). 
that the definition of John domains used in [7] is based on diameter cigar, which is quantitatively equivalent to Definition 1.1 when $E=\mathbf{R}^{n}$. In the same paper, Väisälä proved that if the intersection of two John domains is not too thin then their union is a John domain as the following result shows.

Theorem A. [7, Theorem 3.1] Suppose that $D_{1}$ and $D_{2}$ are c-John domains in $\mathbf{R}^{n}$. If there exist $z_{0} \in D_{1} \cap D_{2}$ and $r>0$ such that $\mathbf{B}\left(z_{0}, r\right) \subset D_{1} \cap D_{2}$ and $d\left(D_{1}\right) \wedge d\left(D_{2}\right) \leq c_{0} r$, where $d\left(D_{i}\right)$ denotes the diameter of $D_{i}(i=1,2)$, then $D_{1} \cup D_{2}$ is a $c^{\prime}$-John domain with $c^{\prime}=2 c\left(c_{0}+1\right)$.

As the first aim of this paper, we study Question 1.1 further. Our result is as follows.

Theorem 1.1. Suppose that both $D_{1}$ and $D_{2}$ are c-John domains in $E$, and that there are $z_{0} \in D_{1} \cap D_{2}$ and $r>0$ such that $\mathbf{B}\left(z_{0}, r\right) \subset D_{1} \cap D_{2}$. If there exists some $r_{1}>0$ such that $r_{1} \leq c_{0} r$ and $D_{1} \subset \mathbf{B}\left(z_{0}, r_{1}\right)$, where $c_{0}>1$ is a constant, then $D_{1} \cup D_{2}$ is a $c^{\prime}$-John domain with $c^{\prime}=c\left(4 c c_{0}+1\right)$.

The proof of Theorem 1.1 will be presented in Section 2. Our proof method is different from that in [7]. Hence when $E=\mathbf{R}^{n}$, we also give a different proof for Theorem A.

We remark that the assumption " $d\left(D_{1}\right) \wedge d\left(D_{2}\right) \leq c_{0} r$ " in Theorem A is equivalent to the statement "at least one of $D_{1}$ and $D_{2}$ is bounded", and the assumption "there exists some $r_{1}>0$ such that $r_{1} \leq c_{0} r$ and $D_{1} \subset \mathbf{B}\left(z_{0}, r_{1}\right)$ " in Theorem 1.1 is equivalent to the statement " $D_{1}$ is bounded". The following example shows that the requirement that "at least one of $D_{1}$ and $D_{2}$ must be bounded" in Theorem 1.1 is necessary.

Example 1.1. Let $D_{1}=\left\{(x, y) \in \mathbf{R}^{2}: x<0\right\}$ and $D_{2}=\left\{(x, y) \in \mathbf{R}^{2}:|y|<\right.$ $x+1\}$. Then both $D_{1}$ and $D_{2}$ are John domains, but $D=D_{1} \cup D_{2}$ is not a John domain.

The proof of Example 1.1 will be given in Section 3 .

Definition 1.2. $D$ is called $c$-uniform in the norm metric in $E$ provided there exists a positive constant $c$ with the property that each pair of points $z_{1}, z_{2}$ in $D$ can be joined by a rectifiable arc $\gamma$ in $D$ satisfying (cf. [5, Section 6.3])

(1) $\ell\left(\gamma\left[z_{1}, z\right]\right) \wedge \ell\left(\gamma\left[z_{2}, z\right]\right) \leq c d(z)$ for all $z \in \gamma$, and

(2) $\ell(\gamma) \leq c\left|z_{1}-z_{2}\right|$.

$D$ is called uniform if it is $c$-uniform for some $c>0$, and $\gamma$ is called a $c$-uniform $\operatorname{arc}$ if it satisfies (1) and (2) (cf. [6, Section 2.16]). See [2, 9] for the generalization of this definition.

As the second aim of this paper, we consider the following question:

Question 1.2. Does Theorem 1.1 hold for uniform domains in E?

The following example shows that even when both $D_{1}$ and $D_{2}$ are bounded uniform domains their union may not be uniform.

Example 1.2. Let $D_{1}=\left\{(x, y) \in \mathbf{R}^{2}:-2<x<1,0<y<2\right\}$ and $D_{2}=$ $D_{3} \cup D_{4}$, where $D_{3}=\left\{(x, y) \in \mathbf{R}^{2}: 0<x<1,-1<y<1\right\}$ and $D_{4}=\{(x, y) \in$ $\left.\mathbf{R}^{2}:-2<x<1,-1<y<0\right\}$. Then both $D_{1}$ and $D_{2}$ are uniform domains, but $D=D_{1} \cup D_{2}$ is not a uniform domain. 
The proof of Example 1.2 will be given in Section 3. For convex and bounded domains in $E$, the following result due to Väisälä, which is from [6].

Theorem B. [6, Theorem 2.19] Suppose that $G$ is a convex domain and that $\mathbf{B}\left(x_{0}, r^{\prime}\right) \subset G \subset \mathbf{B}\left(x_{0}, R^{\prime}\right)$. Then $G$ is $c_{1}$-uniform with $c_{1}=2 \frac{R^{\prime}}{r^{\prime}}$.

In the following we consider Question 1.2 for convex domains and we get

Theorem 1.2. Suppose that $D_{1}$ and $D_{2}$ are convex domains in $E$, where $D_{1}$ is bounded and $D_{2}$ is c-uniform for some $c>1$, and that there exist $z_{0} \in D_{1} \cap D_{2}$ and $r>0$ such that $\mathbf{B}\left(z_{0}, r\right) \subset D_{1} \cap D_{2}$. If there exist constants $R_{1}>0$ and $c_{0}>1$ such that $R_{1} \leq c_{0} r$ and $D_{1} \subset \mathbf{B}\left(z_{0}, R_{1}\right)$, then $D_{1} \cup D_{2}$ is a $c^{\prime}$-uniform domain with $c^{\prime}=(c+1)\left(2 c_{0}+1\right)+c$.

The proof of Theorem 1.2 will be presented in Section 4.

Remark 1.1. Example 1.2 shows that the hypothesis " $D_{2}$ being convex" in Theorem 1.2 is necessary.

\section{The proof of Theorem 1.1}

We show that the theorem holds with $c^{\prime}=c\left(4 c c_{0}+1\right)$. Set $D=D_{1} \cup D_{2}$. Let $x \in D \backslash D_{2}, y \in D \backslash D_{1}, d_{j}(x)=d\left(x, \partial D_{j}\right)$ for $j=1,2$. Then there are John arcs $\alpha \subset D_{1}$ from $x$ to $z_{0}$ and $\beta \subset D_{2}$ from $z_{0}$ to $y$ and an arc $\gamma_{1} \subset \alpha \cup \beta$ from $x$ and $y$. To prove that $\gamma_{1}$ is a $c^{\prime}$-John arc in $D$ it suffices to show that

(1) $\ell(\alpha[x, w]) \leq c^{\prime} d(w)$ for all $w \in \alpha$,

(2) $\zeta(z):=\left(\ell(\alpha)+\ell\left(\beta\left[z_{0}, z\right]\right)\right) \wedge \ell(\beta[z, y]) \leq c^{\prime} d(z)$ for all $z \in \beta$.

We let $x_{0} \in \alpha$ be the point bisecting the length of $\alpha$ and choose $x_{1}$ such that $\ell\left(\alpha\left[x_{1}, z_{0}\right]\right)=\frac{r}{2}$. For any $w \in \alpha$, if $w \in \alpha\left[x, x_{0}\right]$, then we have

$$
\ell(\alpha[x, w])=\ell(\alpha[x, w]) \wedge \ell\left(\alpha\left[w, z_{0}\right]\right) \leq c d_{1}(w) \leq c d(w),
$$

and (1) is proved.

If $w \in \alpha\left[x_{1}, z_{0}\right]$, then

and

$$
d(w) \geq \frac{r}{2} \geq \frac{r_{1}}{2 c_{0}}
$$

which show that

$$
\ell(\alpha)=2 \ell\left(\alpha\left[x, x_{0}\right]\right) \leq 2 c d_{1}\left(x_{0}\right) \leq 2 c r_{1},
$$

$$
\ell(\alpha[x, w]) \leq \ell(\alpha) \leq 2 c r_{1} \leq 4 c c_{0} d(w)
$$

and we obtain (1).

Let $w \in \alpha\left[x_{0}, x_{1}\right]$. Obviously, $\ell(\alpha[x, w]) \wedge \ell\left(\alpha\left[z_{0}, w\right]\right)=\ell\left(\alpha\left[z_{0}, w\right]\right) \geq \frac{r}{2}$, which together with (2.1) show that

$$
\ell(\alpha[x, w]) \leq 2 c r_{1} \leq 4 c \frac{r_{1}}{r} \ell\left(\alpha\left[z_{0}, w\right]\right) \leq 4 c^{2} c_{0} d(w),
$$

which is (1).

The proof of (1) is complete. In the following, we come to prove (2). We let $y_{0} \in \beta$ be the point bisecting the length of $\beta$ and choose $y_{1}$ such that $\ell\left(\alpha\left[z_{0}, y_{1}\right]\right)=\frac{r}{2}$. For any $z \in \beta$, if $z \in \beta\left[y, y_{0}\right]$, then (2) easily follows because $\beta$ is $c$-John in $D_{2}$.

If $z \in \beta\left[y_{1}, z_{0}\right]$, then (2.1) implies that

$$
\zeta(z) \leq \ell(\alpha)+\ell\left(\beta\left[z_{0}, z\right]\right) \leq 2 c r_{1}+c d_{2}(z) \leq 4 c c_{0} d(z)+c d(z)=c\left(4 c_{0}+1\right) d(z),
$$


since $r_{1} \leq c_{0} r \leq 2 c_{0} d(z)$. If $z \in \beta\left[y_{0}, y_{1}\right]$, then we have

$$
\ell(\beta[y, z]) \wedge \ell\left(\beta\left[z, z_{0}\right]\right)=\ell\left(\beta\left[z, z_{0}\right]\right) \geq \frac{r}{2} \geq \frac{r_{1}}{2 c_{0}}
$$

and

$$
\ell(\beta[y, z]) \wedge \ell\left(\beta\left[z, z_{0}\right]\right) \leq c d_{2}(z) \leq c d(z)
$$

which together with (2.1) imply

$$
\zeta(z) \leq \ell(\alpha)+\ell\left(\beta\left[z_{0}, z\right]\right) \leq 2 c r_{1}+c d(z) \leq 4 c^{2} c_{0} d(z)+c d(z)=c\left(4 c c_{0}+1\right) d(z) .
$$

The arbitrariness of $x$ and $y$ shows that $D=D_{1} \cup D_{2}$ is a $c^{\prime}$-John domain with $c^{\prime}=c\left(4 c c_{0}+1\right)$.

\section{The proof of Examples 1.1 and 1.2}

3.1. Proof of Example 1.1. The proof of both $D_{1}$ and $D_{2}$ being John domains easily follows from the fact that an $L$-bilipschitz image of a $c$-John domain is $c^{\prime}$-John with $c^{\prime}=L^{2} c$. Obviously, $z_{0}=\left(-\frac{1}{2}, 0\right) \in D_{1} \cap D_{2}$ and $\mathbf{B}\left(z_{0}, \frac{1}{3}\right) \subset D_{1} \cap D_{2}$. Let $D=D_{1} \cup D_{2}$. Then for any positive integer $n, w_{n}=(-n, 0)$ and $z_{n}=(n, 0) \in D$. For any $\gamma_{n}$ joining $w_{n}$ and $z_{n}$, there must exist a point $u_{n} \in \gamma_{n} \cap\left(D_{1} \cap D_{2}\right)$ such that $d\left(u_{n}\right)<2$ and $\ell\left(\gamma_{n}\left[w_{n}, u_{n}\right]\right) \wedge \ell\left(\gamma_{n}\left[z_{n}, u_{n}\right]\right) \rightarrow \infty$ as $n \rightarrow \infty$. This implies that $D_{1} \cup D_{2}$ is not a John domain.

In order to prove Example 1.2, we introduce the following definition.

Definition 3.1. A domain $D \subset E$ is c-quasiconvex if each pair of points $a, b \in D$ can be joined with an arc $\gamma \subset D$ with

$$
\ell(\gamma) \leq c|a-b|
$$

where $c>1$ is a constant.

Proposition 3.1. If $D \subset E$ is uniform, then it must be quasiconvex.

3.2. Proof of Example 1.2. Theorem B implies that all domains $D_{1}, D_{3}$ and $D_{4}$ are uniform, and Theorem 1.2 shows that $D_{2}$ is also uniform. Obviously, $D=D_{1} \cup D_{2}$ is not quasiconvex, hence by Proposition 3.1, $D$ is not uniform.

\section{The proof of Theorem 1.2}

Before the proof of Theorem 1.2, we introduce two lemmas.

Lemma C. [8, Lemma 3.4] Suppose that $D \subset E$ is a convex domain. The function $d: D \rightarrow \mathbf{R}$ is concave, that is,

$$
d(t a+(1-t) b) \geq t d(a)+(1-t) d(b)
$$

whenever $a, b \in D$ and $t \in[0,1]$.

Lemma 4.1. Suppose that $X$ is a vector space with $\operatorname{dim} X \geq 2$, the vectors $x_{i}$, $y_{i}, z_{i} \in X$ are linearly independent for each $i \in\{1,2\}$, and that

$$
x_{1}-y_{1}=\lambda\left(x_{2}-y_{2}\right), y_{1}-z_{1}=\mu\left(y_{2}-z_{2}\right) \text { and } z_{1}-x_{1}=\tau\left(z_{2}-x_{2}\right)
$$

for constants $\lambda, \mu, \tau$. Then $\lambda=\mu=\tau$. 
Proof. Since

$$
z_{1}-x_{1}=\left(z_{1}-y_{1}\right)+\left(y_{1}-x_{1}\right)=\mu\left(z_{2}-y_{2}\right)+\lambda\left(y_{2}-x_{2}\right),
$$

we know

$$
(\tau-\lambda) x_{2}+(\lambda-\mu) y_{2}+(\mu-\tau) z_{2}=0 .
$$

By the linear independence of $\left\{x_{2}, y_{2}, z_{2}\right\}$ we get $\lambda=\mu=\tau$.

Now we are ready to prove Theorem 1.2.

Proof of Theorem 1.2. We show that the theorem holds with $c^{\prime}=(c+1)\left(2 c_{0}+\right.$ $1)+c$. Set $D=D_{1} \cup D_{2}$ and $D_{0}=D_{1} \cap D_{2}$. Let $a \in D \backslash D_{2}, b \in D \backslash D_{1}, z_{1}=\frac{a+b}{2}$ and $s=\frac{|a-b|}{2}$.

Case 4.1. $\left|z_{1}-z_{0}\right| \leq 2 c_{0} s$. There is a $c$-uniform arc $\beta \subset D_{2}$ from $z_{0}$ to $b$ and an arc $\gamma \subset\left|a-z_{0}\right| \cup \beta$ from $a$ to $b$. To prove that $\gamma$ is $c^{\prime}$-uniform in $D$ it suffices to show that

(1) $\left|a-z_{0}\right|+\ell(\beta) \leq c^{\prime}|a-b|$,

(2) $|a-x| \leq c_{0} d(x)$ for all $x \in\left[a, z_{0}\right]$,

(3) $\zeta(y):=\left(\left|a-z_{0}\right|+\ell\left(\beta\left[z_{0}, y\right]\right)\right) \wedge \ell(\beta[b, y]) \leq c^{\prime} d(y)$ for all $y \in \beta$.

Since $\left|a-z_{0}\right| \leq\left|a-z_{1}\right|+\left|z_{1}-z_{0}\right| \leq s+2 c_{0} s$ and similarly $\left|b-z_{0}\right| \leq s+2 c_{0} s$, we have

$$
\left|a-z_{0}\right|+\ell(\beta) \leq\left(2 c_{0}+1\right) s+c\left|b-z_{0}\right| \leq(c+1)\left(2 c_{0}+1\right) s \leq c^{\prime}|a-b|,
$$

and (1) is proved.

If $x \in\left[a, z_{0}\right]$, then $x=(1-t) a+t z_{0}$ for some $t \in[0,1]$, and we have $|a-x|=$ $t\left|a-z_{0}\right| \leq t c_{0} r$. Lemma $\mathrm{C}$ implies that

$$
d(x) \geq d_{1}(x) \geq(1-t) d_{1}(a)+t d_{1}\left(z_{0}\right) \geq t d_{1}\left(z_{0}\right) \geq t r .
$$

As $|a-x|=t\left|a-z_{0}\right| \leq t c_{0} r$, this yields (2).

Let $y \in \beta$ and let $y_{0} \in \beta$ be the point bisecting the length of $\beta$. If $y \in \beta\left[y_{0}, b\right]$, then the $c$-uniformity of $\beta$ gives $\zeta(y)=\ell(\beta[y, b]) \leq c d_{2}(y)$. If $y \in \beta\left[z_{0}, y_{0}\right]$, then

$$
\zeta(y) \leq\left|a-z_{0}\right|+\ell\left(\beta\left[z_{0}, y\right]\right) \leq c_{0} r+c d_{2}(y) \leq c_{0} d\left(z_{0}\right)+c d(y) .
$$

Here $d\left(z_{0}\right) \leq d(y)+\left|z_{0}-y\right| \leq d(y)+\ell\left(\beta\left[z_{0}, y\right]\right) \leq(1+c) d(y)$, and we obtain (3).

Case 4.2. $\left|z_{1}-z_{0}\right|>2 c_{0} s$. Set $e=(b-a) /|b-a|$ and $a_{0}=z_{0}+r e$. As $a \notin D_{2}$ and $b \notin D_{1}$, these points do not lie on the line through $z_{0}$ and $z_{1}$. Hence there is a unique point $w \in\left[z_{0}, z_{1}\right] \cap\left[a_{0}, a\right]$. Applying Lemma 4.1 to the triples $\left(w, z_{0}, a_{0}\right)$ and $\left(w, z_{1}, a\right)$ we get $\left|w-z_{1}\right|=s\left|w-z_{0}\right| / r$. Replacing $a$ and $a_{0}$ by $b$ and $b_{0}=z_{0}-r e$, respectively, we see that $w \in\left[z_{0}, z_{1}\right] \cap\left[b_{0}, b\right]$. Hence $w \in D_{0}$, which implies that $\left[z_{0}, w\right] \subset D_{0}$. Since $w \in D_{1} \subset \mathbf{B}\left(z_{0}, c_{0} r\right)$, we have $\left|w-z_{0}\right| \leq c_{0} r$, whence $\left|w-z_{1}\right| \leq c_{0} s$.

Set $u=\left(z_{1}-z_{0}\right) /\left|z_{1}-z_{0}\right|$ and $y_{1}=w-c_{0} s u$. Then $\left|y_{1}-z_{1}\right|=\left|y_{1}-w\right|+\left|w-z_{1}\right| \leq$ $2 c_{0} s$, whence $y_{1} \in\left[z_{0}, w\right]$. There is a $c$-uniform arc $\beta_{1} \subset D_{2}$ from $y_{1}$ to $b$ and an arc $\gamma \subset\left[a, y_{1}\right] \cup \beta_{1}$ from $a$ to $b$. To prove that $\gamma$ is $c^{\prime}$-uniform in $D$ it suffices to show that

(1) $|a-y|+\ell\left(\beta_{1}\right) \leq c^{\prime}|a-b|$,

(2) $|a-x| \leq\left(2 c_{0}+1\right) d(x)$ for all $x \in\left[a, y_{1}\right]$,

(3) $\zeta_{1}(y):=\left(\left|a-y_{1}\right|+\ell\left(\beta_{1}\left[y_{1}, y\right]\right)\right) \wedge \ell\left(\beta_{1}[b, y]\right) \leq c^{\prime} d(y)$ for all $y \in \beta_{1}$. 
We have

$$
\left|a-y_{1}\right| \leq\left|a-z_{1}\right|+\left|z_{1}-y_{1}\right| \leq s+2 c_{0} s, \quad \ell\left(\beta_{1}\right) \leq c\left|b-y_{1}\right| \leq c\left(2 c_{0}+1\right) s,
$$

and (1) follows.

If $x \in\left[a, y_{1}\right]$, then $x=(1-t) a+t y_{1}$ for some $t \in[0,1]$. It follows from Lemma $\mathrm{C}$ that

$$
d(x) \geq d_{1}(x) \geq(1-t) d_{1}(a)+t d_{1}\left(y_{1}\right) \geq t d_{1}\left(y_{1}\right)
$$

As $\left|w-z_{0}\right| \leq c_{0} r$, we similarly obtain

Hence

$$
d_{1}\left(y_{1}\right) \geq \frac{c_{0} s}{\left|w-z_{0}\right|} d_{1}\left(z_{0}\right) \geq \frac{c_{0} s r}{\left|w-z_{0}\right|} \geq s .
$$

which is (2).

$$
|a-x|=t\left|a-y_{1}\right| \leq t\left(2 c_{0}+1\right) s \leq\left(2 c_{0}+1\right) d(x),
$$

Let $y \in \beta_{1}$ and $y_{0} \in \beta_{1}$ be the point bisecting the length of $\beta_{1}$. If $y \in \beta_{1}\left[y_{0}, b\right]$, then (3) follows from the $c$-uniformity of $\beta_{1}$ in $D_{2}$. Let $y \in \beta_{1}\left[y_{1}, y_{0}\right]$. Now $(2)$ and the $c$-uniformity of $\beta_{1}$ imply that

$$
\zeta_{1}(y) \leq\left|a-y_{1}\right|+\ell\left(\beta_{1}\left[y_{1}, y\right]\right) \leq\left(2 c_{0}+1\right) d\left(y_{1}\right)+c d_{2}(y) .
$$

Here $d\left(y_{1}\right) \leq d(y)+\left|y-y_{1}\right| \leq d(y)+\ell\left(\beta_{1}\left[y_{1}, y\right]\right) \leq(c+1) d(y)$, and we obtain (3).

The proof of Theorem 1.2 is complete.

Acknowledgements. The authors thank the referee very much for his or her careful reading of this paper and many valuable suggestions.

\section{References}

[1] John, F.: Rotation and strain. - Comm. Pure. Appl. Math. 14, 1961, 391-413.

[2] Klén, R., S. K. SAhoo, and M. Vuorinen: Uniform continuity and $\varphi$-uniform domains. arXiv: 0812.4369v3 [Math. MG].

[3] Martio, O., and J. Sarvas: Injectivity theorems in plane and space. - Ann. Acad. Sci. Fenn. Ser. A I Math. 4, 1978, 383-401.

[4] NÄkKI, R., and J. VÄIsÄLÄ: John disks. - Expo. Math. 9, 1991, 3-43.

[5] VÄıs̈̈LÄ, J.: Free quasiconformality in Banach spaces. II. - Ann. Acad. Sci. Fenn. Ser. A I Math. 16, 1991, 255-310.

[6] VÄısÄlä, J.: Relatively and inner uniform domains. - Conform. Geom. Dyn. 2, 1998, 56-88.

[7] VÄIsÄLÄ, J.: Unions of John domains, - Proc. Amer. Math. Soc. 128, 1999, 1135-1140.

[8] VÄıs̈̈LÄ, J.: Quasihyperbolic geodesics in convex domains. - Results Math. 48, 2005, $184-195$.

[9] Vuorinen, M.: Conformal invariants and quasiregular mappings. - J. Analyse Math. 45, 1985, 69-115. 